\title{
Prediction of the spatial variability of coal- bearing rocks at the Elginsky coal mine
}

\author{
Yuri Malinin ${ }^{1 *}$, Nikolai Grib ${ }^{1,5}$, Pavel Kuznetsov ${ }^{1}$, Zhang Ze $\mathrm{Z}^{2,3,4}$ \\ ${ }^{1}$ Technical Institute (branch) of the M.K. Ammosov North-Eastern Federal University, 16 \\ Kravchenko str., Neryungri, Republic of Sakha (Yakutia), 678960, Russia \\ ${ }^{2}$ State Key Laboratory of Frozen Soil Engineering, Northwest Institute of Eco-Environment and \\ Resources, Chinese Academy of Sciences, Lanzhou 730000, China. \\ ${ }^{3}$ School of Civil Engineering \& Institute of Cold Regions Engineering, Science and Technology, \\ Northeast Forestry University, Harbin 150040, China. \\ ${ }^{4}$ Northeast-China Observatory and Research-Station of Permafrost Geo-Environment, Ministry of \\ Education, Northeast Forestry University, Harbin 150040, China \\ ${ }^{5}$ Academy of Sciences of the Republic of Sakha (Yakutia), 677007, Russia
}

\begin{abstract}
The article presents a brief analysis of the key methods used for spatial modelling of mining and geological indicators describing the composition, structure and state of rock deposits. The main limitations of the analysed methods when applied under real conditions are outlined. It is proposed to overcome these limitations using Markov nonlinear algorithms. By applying the principles of multi-dimensional Markov modelling to a geological object, interval types were determined for modelling mining and geological parameters of the Elginsky coal mine. As an example, the article presents the results of predicting the ash content for the U5 section of the Elginsky coal mine on the basis of one of the crosssections of the developed three-dimensional model.
\end{abstract}

\section{Introduction}

The methods of spatial modelling are widely used in mining and geological research for creating accurate models of the composition, structure and state of rock massifs (RMs). In practice, RMs are spatially represented using the graphical images of sections along observation lines. Such sections are constructed using data derived from the plane of observation lines, such as wells located along exploration lines, seismic profiles, electrical survey lines, etc. In essence, the as-constructed sections comprise 2-dimensional models.

The widespread application of modern computing technologies in geological exploration practice has required the development of new methodological approaches to provide for the most efficient use of electronic facilities when modelling RMs.

One of the challenges arising in predicting the spatial variability of parameters in coal deposits involves the creation of interpolation 3-D models, which can represent adequately the distribution of the main properties of geological objects. This issue is currently addressed using the following two approaches. In the first case, the volume of a geological body is divided into an array of planes, for each of which the observed values are re-

*Corresponding author: alkor.05@mail.ru 
calculated into the nodes of a regular grid [1]. The set of planes corresponds to the 3dimensional distribution of a particular parameter. Another approach involves determining the coefficients of polynomial regression equations in Cartesian coordinates. A significant drawback of these both approaches is the assumption that the properties of the array under study are varying continuously (the function is differentiable at all points of the volume). However, it is common knowledge that geological bodies are inherently divided into layers, both stratigraphic (layers of various lithotypes) and tectonic (disturbances). Therefore, the continuity of variations in properties can only be considered as a special case. When solving geological problems, one should assume the fundamental non-linearity of geological properties. A popular mathematical approach that can be used in such cases is Markov nonlinear algorithms [5-10].

In this work, we develop an algorithm for Markov N-dimensional modelling on the basis of an approach implying the construction of a probabilistic image of geological bodies on an N-dimensional grid, rather than assigning coordinate points of space to the values of geological and geophysical indicators [5-7]. The creation of a probabilistic image is carried out on a training set, in which the space coordinates of points are considered as those of the phase space, a sequence of numerical values that are presented in the form of a Markov sequence with a strictly defined number and sequence of steps. For the unambiguous localization of points in the phase space, all the variants of transitions (permutations) of the coordinate axes $\mathrm{X}, \mathrm{Y}, \mathrm{Z}$, and $\mathrm{T}$ (transposed vector) are used. The number of such combinations is determined by the formula:

$$
N=n \times n-n,
$$

where $N$ is the number of variables in the vector; $n$ is the number of initial variables included in the vector.

Thus, under 2, 4 and 5 initial variables, the vector will consist of 3, 12 and 20 variables, respectively. Notably, such an increase in the number of vector variables does not affect the size of the matrices of transition probabilities, since the number of states that determine the size of the matrices does not increase. The use of sequences of this type increases the recognition efficiency for nominal variables rather significantly.

A successive accumulation of coordinate vectors (sequences of values) produces matrices of accumulated frequencies describing a specific value or a range of values (class) of the geological parameter under modelling. Further transformation of the obtained frequency matrices into transition probabilities matrices creates a model representing the distribution of the analysed parameter in the coordinates of the N-dimensional space.

An essential aspect in the formalization (abstraction) of parameters and variables for the purpose of using their mathematical properties and relations consists in the introduction of a variable (parameter) representing the image of an individual property. The observation channel, through which the ai property is represented by the $V i$ variable, is implemented by the function:

$$
\text { ai: } A i \Rightarrow V i
$$

This function is homomorphic with respect to the assumed properties of the Ai and $V i$ sets. For some parameters and variables, observation channels comprise explicitly specified ai functions; for others, the function should be specified by the researcher. In other cases, a set of properties can be distinguished into the groups corresponding to subsets bounded by the boundaries when the parameter scale is evenly divided. Other methods for creating the image of a property are also possible. The approach involving division into classes limits their number by the magnitude of the observation error, the volume and density of distribution across the scale of variable states.

The next step is to assign a code value to the variable under study in order to indicate both its state and its label. This is achieved by assigning an integer value in the interval (1... 
t) to this variable. The next variable is assigned a value in the interval $(t+1, \ldots n)$, etc. Thus, the label is an interval, and the state of the variable is a number in this interval.

In comparison with distinct channels used for observing variables and parameters, the model in this paper uses uncertain (fuzzy) channels, for which an appropriate representing function is introduced. In this case, distinct channels will be considered as a special case of fuzzy channels. The fundamental difference between distinct and fuzzy observation channels is the following: in the former case, the probability of a transition is related to the modal value of the class; in the latter - by the boundaries of the class. The variables and parameters transformed in the described way create the mathematical image of a system, the further study of which will be performed using the Markov chains of integer values for variables with branching values for fuzzy channels.

Markov recognition - classification. There is an alphabet $\mathrm{A}=\{$ ai $\}$ and its division into classes $B=\{b j=\{a i j\}\}$. It is known that, in the $C=(c 1, c 2, \ldots)$ chain of symbols from $A$, the alternation of classes obeys the 1st order Markov process with the alternation matrix $\mathrm{M}$ $=\{\mathrm{Pkl}\}$. The task is to classify the elements of $\mathrm{C}$ such that the entropy of the chain over $\mathrm{M}$ is maximized. The Markov linkage algorithm itself is a kind of dynamic programming algorithm that maximizes the objective function $C$ on the set of chains $\left\{L=\left(l_{1}, l_{2}, \ldots \ln \right)\right\}$, where $l i$ is a cluster defined on the set of Markov chains.

It is proposed to use three types of intervals for the parameter under modelling:

- an interval represented by one lithotype;

- an interval of rocks with similar physical characteristics;

- a section interval typified on the basis of a set of Markov characteristics (lithological or geophysical).

The recognition is carried out by points in the phase (Cartesian) space. Point coordinates are generated according to the section planes selected by the researcher. The boundary points of the coordinates and the sampling step are set. As the result, a probabilistic estimate for the distribution of geological and geophysical characteristics of the model on the plane of an RM section or a sequence of sections in the selected time interval is generated. The forecast is visualized on a display in two-dimensional coordinates.

The described algorithm was tested using prototypes of Markov multifactor forecasting programs on distinct channels when assessing the quality indicators of coals [11-17]. Figure 1 shows an example of a coal deposit section. The coal seam U5 of the Elginsky coal deposit is represented by a change in the ash content along one of the sections (U44-U44). The changes in the ash content used in the construction were determined in separate layer intersections by laboratory methods. Using a simulation program, a 3-dimensional model of changes in the ash content was calculated. Setting $Y=44$, the values of $X$ (across the value range of 90-120) and $Z$ (across the value range of $0-14$ ) were sequentially generated. For the sake of convenience, the obtained data are presented by the numerical values of ash content. Thus, the fundamental possibility of a three-dimensional modelling of RMs using the potential of nonlinear Markov statistics has been demonstrated.

It should be noted that the proposed technique is characterized by the following important feature. Each layer identified in the interval of a coal seam was considered as an independent geological body. Similarly, the underlying and upper layers, including coal ones, were considered as independent geological bodies genetically related to the layer under study. Such an assumption is necessary, since thick coal seams of a complex structure exhibit a significant variability in petrophysical characteristics as a result of changing formation conditions. The criterion for dividing the coal seam into layers is the change in ash content, which manifests itself on geophysical diagrams, as well as the presence of interlayers with a thickness of above $0.10 \mathrm{~m}$. Thus, several layers can be distinguished in a geological body, with their number varying depending on the RM area 
and a layer-by-layer correlation being impossible due to the significant variability of the elements constituting the RM.

\section{Tomogram on the line $\mathrm{X}$ 90-90}

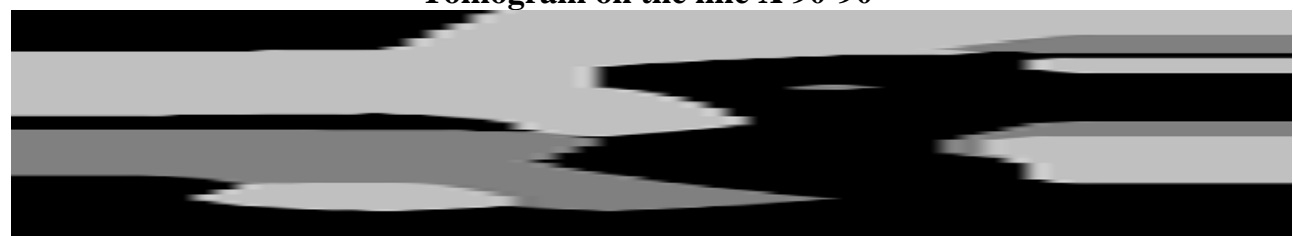

Tomogram on the line X 95-95

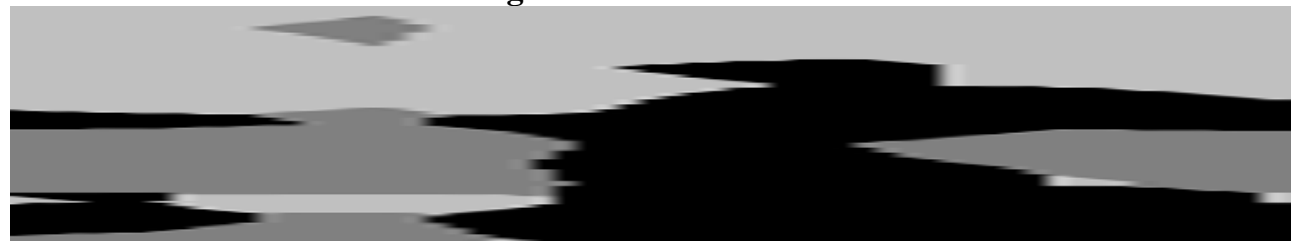

Tomogram on the line X 100-100

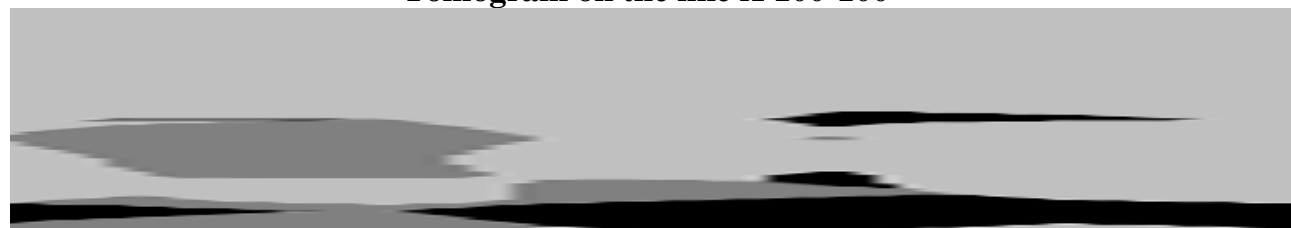

\section{Tomogram on the line X 105-105}

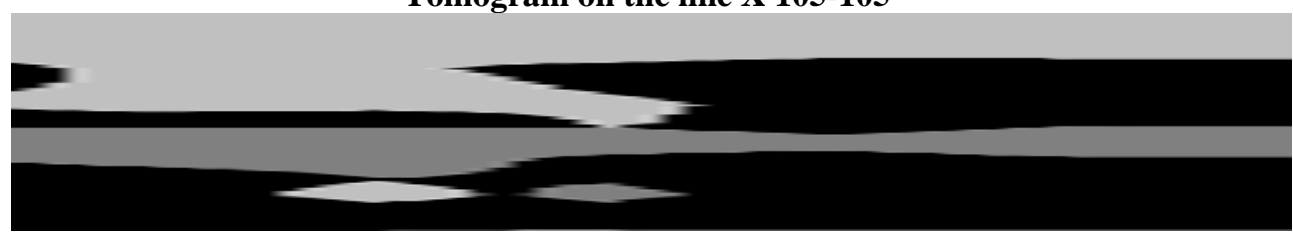

Tomogram on the line X 110-110

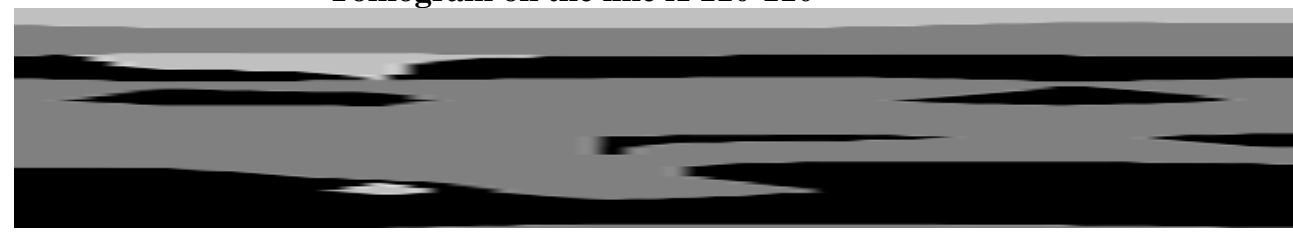

Tomogram on the line X 115-115

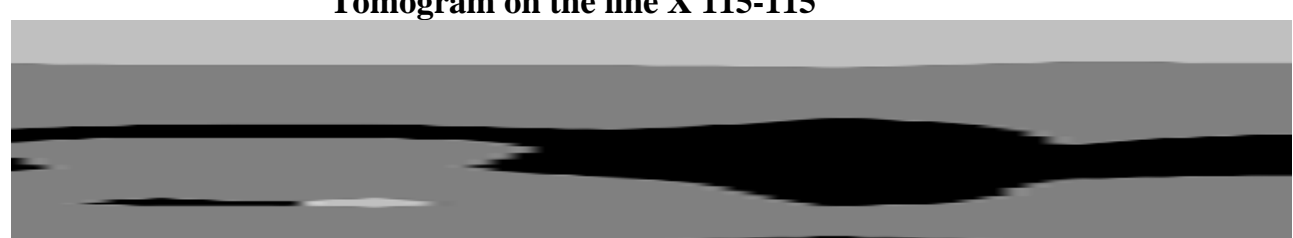

Tomogram on the line $X$ 120-120

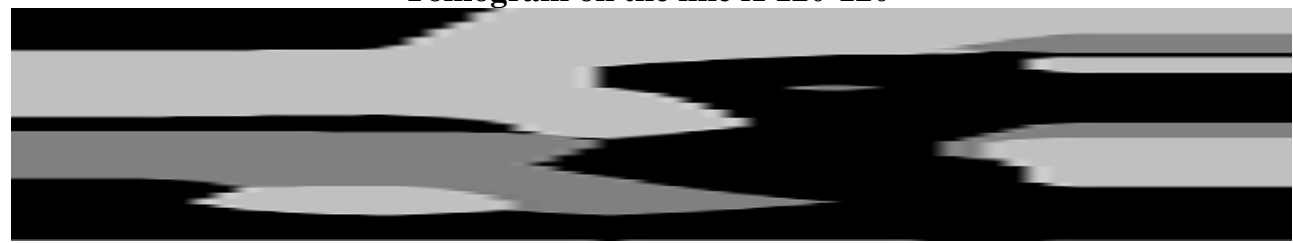

Fig.1. Sections of the U5 coal seam of the Elginsky coal deposit (changes in the ash content along the U44-U44 section) 
In this regard, it became necessary to construct 3-dimensional models representing the variability of quality indicators, reflecting both lateral and vertical components. Due to the high information content of such models, their construction requires significant time and effort. The construction of a 3-d model for a section of the U5 seam located in the SouthEastern part of the Elginsky coal deposit was carried out. The U5 seam lying at absolute elevations from 40 to $400 \mathrm{~m}$ is characterized by a thickness of 7 to 14 meters and the ash content of coal packs from 8 to $38 \%$.

For each sub-section of this seam crossed with a well, both within and beyond the boundaries of the selected area, a per-pack forecast of ash content was given in accordance with the method of batch Markovian modelling [5-7]. In addition to genetic factors, the model considered the influence of the hypergenesis zone and permafrost. For reservoir intersections, graphs of changes in the ash content along the section were constructed.

Figure 1 shows an example of a section of the U5 coal-bearing layer at the Elginsky coal mine, demonstrating changes in the ash content along one of the sections (U44-U44). The changes in the ash content used in the construction were determined in separate layer intersections by laboratory methods. Using a simulation program, a 3-dimensional model of changes in the ash content was calculated. Setting $Y=44$, the values of $X$ (across the value range of 90-120) and $Z$ (across the value range of 0-14) were generated. In this figure, coal is depicted in black; coal rocks - in dark grey; rocks - in light grey. Thus, the fundamental possibility of three-dimensional modelling of the RM using a mathematical apparatus of nonlinear Markov statistics has been demonstrated.

\section{References}

1. N. N. Grib, P. Yu. Kuznetsov, M. V. Tereshchenko, E.F. Redlich. Trends in the development of science and education, 44(5), 90 (2018)

2. P. Yu. Kuznetsov, N. N. Mushroom, G. V. Mushroom. VSU Bulletin. Series: Geology, 1, 82 (2019)

3. N. N. Grib, A. A. Syasko, A. V. Kachaev, P. Yu. Kuznetsov, M. V. Tereshchenko. Coal, 10, 79 (2016)

4. $\quad$ M. Khandelwal. Pure Appl. Geophys, 4(170), 507 (2013)

5. O. L. Momolkin. Mathematical modeling of coal quality indicators (on the example of Neryungri coal deposit) (Prague, Vêdecko vyavatelské centrum "Sociosféra CZ") (2016)

6. N. N. Grib, A. V. Samokhin, A. G. Chernikov. Methodological foundations of a systematic study of a rock mass (Yakutsk, YaNTs SB RAS) (2000)

7. A. G. Chernikov. Recommendations for the processing and reinterpretation of geological and geophysical data based on automated geological and mathematical modeling of the properties, composition, structure of coal-bearing deposits in the South Yakutia basin. (Moscow, Geonauka) (1990)

8. A. B. Vistellius. Fundamentals of Mathematical Geology. (Leningrad, Science). (1980)

9. V. V. Grechukhin. Study of coal formations by geophysical methods. (Moscow, Nedra) (1980)

10. N. N. Moiseev. Mathematical problems of system analysis. (Moscow, URSS) (2011)

11. M. V. Tereschenko, N. N. Grib, K. Mackey IEJME - Mathematics Education, 8 (11), 2975

12. N. N. Grib, P. Yu. Kuznetsov, A. A. Syasko, A.V. Kachaev. Modern problems of science and education, 2, (2013) 
13. P. Yu. Kuznetsov, N.. Mushroom. Proceedings of universities. Mining Journal, 5, 22 (2018)

14. N. N Grib, P. Yu. Kuznetsov Coal, 1, 68 (2018)

15. N. N. Grib, A. A. Syasko, D. N. Grib, P. Yu. Kuznetsov, A. V. Kachaev. Coal, 12, 43 (2017)

16. D. V. Yakovlev, A. G. Chernikov, V. S. Coal, 12, 19 (1991)

17. P. Yu. Kuznetsov, N. N. Mushroom, Yu. N. Skomoroshko. Mining Journal, 3, 47 (2017) 